\section{Gentest kann Therapieentscheidung bei Mammakarzinompatientinnen revidieren}

\begin{abstract}
Um die Rückfallwahrscheinlichkeit bei Patientinnen mit frühem Hormonrezeptor-positivem Mammakarzinom abzuschätzen, werden Multi-Gen-Tests eingesetzt. Eine deutsche Multicenterstudie untersuchte den Einfluss des Oncotype DX ${ }^{\circledR}$-Tests auf die Wahl der adjuvanten Therapie.
\end{abstract}

$\mathrm{M}_{\mathrm{t}}^{\mathrm{i}}$ it dem Oncotype $\mathrm{DX}^{-}$-Brustkrebstest kann die Aktivität von 21 Krebsund Kontrollgenen im Tumorgewebe von Frauen mit Östrogen-Rezeptor (ER)-positivem Mammakarzinom bestimmt werden. Der so ermittelte Recurrence-Score (RS) bietet eine zusätzliche Entscheidungshilfe für oder gegen eine adjuvante Chemotherapie. Der Einsatz des MultiGen-Tests wird in mehreren internationalen Leitlinien (z. B. ASCO) empfohlen.

In einer prospektiven Studie an $379 \mathrm{~Pa}-$ tientinnen mit ER-positivem, HER2-negativem Mammakarzinom ( $\mathrm{N} 0$ und $\mathrm{N}+$ ) im frühen Stadium wurde der Einfluss des RS auf die Entscheidungen zur adjuvanten Behandlung untersucht. Ausgewertet wurden die Daten von 366 Patientinnen, davon 244 nodal negativ und 122 positiv. Bei $33 \%$ aller Patientinnen veränderten die Oncotype-Ergebnisse die weiteren Therapieentscheidungen (N0 30\%, $\mathrm{N}+39 \%$ ): Bei $38 \%$ aller Patientinnen (N0 $39 \%, N+37 \%$ ) wurde eine initiale Empfehlung zur Chemo- plus Hormontherapie nach Kenntnis des RS in Richtung endokrine Therapie verändert, bei $25 \%$ (N0 $22 \%, N+39 \%)$ mit einer initialen Empfehlung für eine endokrine Monotherapie veränderte der RS die Empfehlung in
Richtung chemoendokriner Behandlung. Der mithilfe eines Fragebogens ermittelte Score zum Therapieentscheidungskonflikt der Patientinnen verbesserte sich durch die RS-Ergebnisse um $6 \%(\mathrm{p}=$ $0,028)$ und die per Fragebogen ermittelte Zuversicht des Arztes um 45\% ( $<<0,001)$. Der Einsatz des 21-Gen-Tests ist im Vergleich zur bisherigen klinischen Praxis ohne Gentestung kostensparend.

Fazit: Der per Multi-Gen-Test ermittelte Recurrence-Score verändert bei einem nicht unerheblichen Anteil von Frauen mit frühem ER-postivem Brustkrebs die adjuvante Therapieentscheidung. Durch den Test können Therapiekosten eingespart werden.

Barbara Kreutzkamp

Eiermann W et al. The 21-gene recurrence score assay impacts adjuvant therapy recommendations for ER-positive, node-negative and nodepositive early breast cancer resulting in a riskadapted change in chemotherapy use. Ann Oncol. 2013;24(3):618-24.

\title{
Prämenopausale Frauen mit chemotherapieinduzierter Amenorrhö bei Brustkrebs: Vorsicht bei Umstellung auf Aromataseinhibitoren
}

Bei prämenopausalen Mammakarzinompatientinnen mit einer Chemotherapieinduzierten Amenorrhö können Aromataseinhibitoren (Als) die Ovarfunktionen wieder anregen. In einer Studie wurden Prädiktoren und die Auswirkungen überprüft.

A romataseinhibitoren sind Bestandteile der adjuvanten Hormontherapie bei postmenopausalen, Hormonrezeptor-positiven Brustkrebspatientinnen. Bei prämenopausalen Frauen, die eine chemotherapieinduzierte Amenorrhö (CIA) entwickelt haben, wird Tamoxifen für den Beginn der adjuvanten Hormontherapie empfohlen. In welchem Ausmaß und mit welchen Folgen eine Umstellung auf einen AI die Ovarfunktion wieder ankurbelt, wurde in einer prospektiven Studie untersucht.

Einbezogen wurden 53 Patientinnen mit einem Östrogenrezeptor (ER)-positiven Mammakarzinom unter einer Tamoxifen-Therapie, die seit mindestens zwei Jahren eine Chemotherapieinduzierte Amenorrhö und E2-Spiegel auf postmenopausalem Niveau hatten. Die Patientinnen wurden auf den AI Exemestan umgestellt und das Wiederauftreten der ovariellen Funktionen wurde biochemisch und klinisch untersucht.

In den indirekten und direkten E2Messungen zeigten sich vergleichbare Inzidenzen für die Wiederherstellung der Ovarfunktion (32 bzw. $30 \%$ ) sowie vergleichbare Zeiten bis zur erneuten ovariellen Funktion (5,4 bzw. 6,0 Monate). In multivariaten Analysen erwies sich lediglich das Alter, in dem auf Exemestan umgestellt wurde, als Marker für die Wahrscheinlichkeit einer angekurbelten Ovarfunktion. Patientinnen mit wieder angeregter Ovarfunktion wiesen gegenüber denen ohne signifikant höhere E2-Spiegel auf ( $\mathrm{p}=0,001)$. Das krankheitsfreie Überleben war dagegen signifikant vermindert $(p=0,04)$.

Fazit: Brustkrebspatientinnen mit einer chemotherapieinduzierten Amenorrhö sollten nur dann von Tamoxifen auf Exemestan umgestellt werden, wenn sie älter als 48 Jahre sind. Bei jüngeren Frauen mit einem unter dem AI aufgetretenen E2-Anstieg ist die Rückfallgefahr höher.

Barbara Kreutzkamp

Guerrero A et al. Incidence and predictors of ovarian function recovery (OFR) in breast cancer (BC) patients with chemotherapy-induced amenorrhea (CIA) who switched from tamoxifen to exemestane. Ann Oncol. 2013;24(3):674-9.

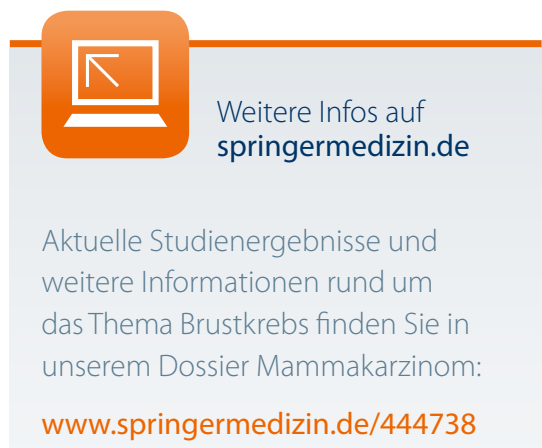

